\title{
Modélisation d'un tube à onde progressive pour la caractérisation de matériaux acoustiques de masquage
}

\author{
G. BESSARD, G. HAW, P. BIGOTTE, R. BOSSUT, B. HAMONIC, J.-N. DECARPIGNY et \\ C. GIANGRECO*
}

I.E.M.N., UMR 9929 du CNRS, Département ISEN, 41 Boulevard Vauban, 59046 Lille cedex, France

${ }^{*}$ DCN/ING/SUD, Département LSM, Le Brusc, 83140 Six-Fours-les-Plages, France

\begin{abstract}
Résumé : Afin de caractériser des matériaux de masquage en basse fréquence en mesurant leur efficacité de masquage, un tube à onde progressive a été développé. Cette communication décrit brièvement le formalisme utilisé ainsi que le modèle éléments finis employé pour simuler le comportement du tube à onde progressive.
\end{abstract}

Abstract: To characterize decoupling materials, at low frequencies, by measuring their decoupling efficiency, a progressive wave duct has been developed. This communication describes briefly the formalization used, and the finite element model used to simulate the progressive wave duct behaviour.

\section{INTRODUCTION.}

La connaissance des caractéristiques acoustiques des matériaux de masquage utilisés pour des applications sous-marines du type barrière acoustique est utile pour le concepteur de système sonar. Le coefficient de masquage (CM) est determine à la DCN/ING/SUD/LSM par la mesure des coefficients de réflexion et de transmission du panneau à caractériser. Ce panneau est situé dans une cuve anéchoïque [1]. La gamme de fréquence actuelle utilisée par cette méthode varie de $1500 \mathrm{~Hz}$ à $20 \mathrm{kHz}$. Afin de compléter la caractérisation dans le domaine des basses fréquences en dessous de $1500 \mathrm{~Hz}$, une méthode de mesure directe du coefficient de masquage utilisant un tube à onde progressive est employée. Dans le premier paragraphe, le principe de la mesure, la description du montage expérimental et celle du modèle éléments finis sont décrits. Le deuxième paragraphe présente la validation du principe de mesure et les premiers résultats obtenus.

\section{PRINCIPE ET DESCRIPTION DE LA MESURE.}

Le coefficient de masquage indique la capacité du matériau à réduire le bruit rayonné dans l'eau par la structure sous-marine qui le supporte. En supposant que la structure vibre uniformément, $C$. Audoly [1] définit ce coefficient comme le rapport entre la pression émise par la structure avec le matériau de masquage $(S)$ et la pression obtenue sans le matériau $\left(\mathrm{S}_{0}\right)$ voir figure 1 : 


$$
\mathrm{CM}=\frac{\mathrm{S}}{\mathrm{S}_{0}}
$$

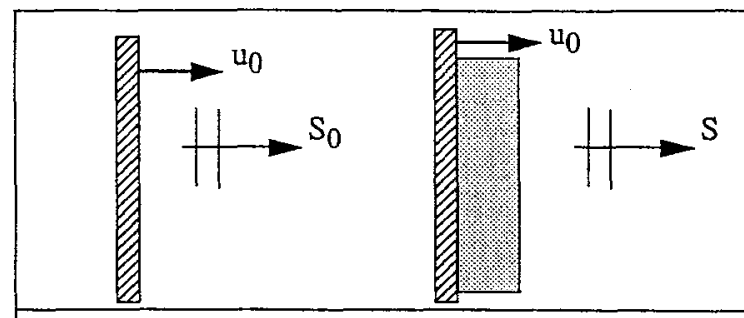

Figure $1 \mathrm{a}$ : sans materiau Figure $1 \mathrm{~b}$ : avec matériau

Le modèle théorique développé par C.Audoly [1] permet à partir de la mesure du coefficient de réflexion $r$ et de transmission $t$ du panneau de déterminer le coefficient de masquage CM par la relation suivante : avec:

$$
\mathrm{CM}=\frac{\mathrm{te} \mathrm{e}^{\mathrm{ikh}}}{1-\mathrm{r}} \quad \text { (2) } \begin{aligned}
& \mathrm{t}=\text { coefficient de transmission complexe } \\
& \mathrm{k}=\text { nombre d'onde } \\
& \mathrm{h}=\text { épaisseur du panneau }
\end{aligned}
$$

Cette relation est valable dans le cas d'un panneau retournable et de dimensions infinies dans les directions perpendiculaires à la direction de propagation de l'onde incidente, elle l'est également pour un panneau non symétrique [2]. Plus CM est petit devant un plus le matériau est efficace. Si le module de $\mathrm{CM}$ est égal à un alors le matériau est considéré comme inefficace pour une application de masquage. Le laboratoire d'acoustique de I' ISEN a mis au point un tube à onde progressive rempli d'eau pour caractériser des hydrophones cardioïdes à gradient de pression [3]. L'utilisation de cette installation autorise une étude dans le domaine des basses fréquences, c'est-à-dire entre $200 \mathrm{~Hz}$ et $3000 \mathrm{~Hz}$. Le but de cette expérience est d'obtenir le coefficient de masquage directement à partir de la relation (1). Pour cela, le tube est composé de quatre tronçons en acier de $600 \mathrm{~mm}$ chacun, son rayon interne est de $96 \mathrm{~mm}$ et son épaisseur est égale à $16 \mathrm{~mm}$. Le dispositif expérimental est présenté sur la figure 2.

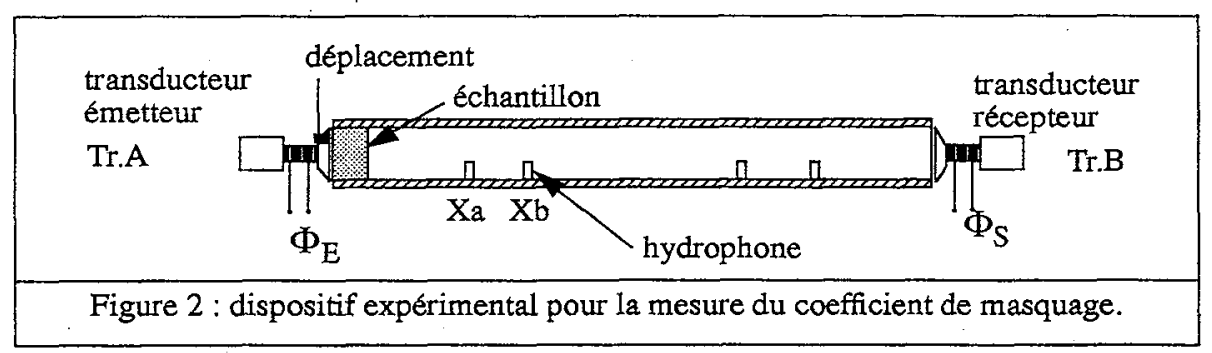

Dans ce montage, l'échantillon à caractériser est collé sur le pavillon du transducteur émetteur (Tr.A). Cet ensemble ferme une des extrémités du tube. A l'autre extrémité, le tube est fermé par le deuxième transducteur dit "récepteur" (Tr.B). Le tube est ensuite rempli d'eau et une pression hydrostatique de deux bars est appliquée.

A partir de l'émission sonore générée par le transducteur émetteur, excité à un Volt, une onde stationnaire est créée dans la cavité fluide. Le transducteur récepteur est commandé par une tension, cette tension est calculée à partir de la valeur du coefficient de réflexion r' dans le fluide qui rend compte du taux d'onde stationnaire. Le coefficient $r$ ' est obtenu à partir de la mesure de la pression entre les 
hydrophones Xa et Xb situés sur la génératrice du tube. Un algorithme de calcul de la tension complexe permet de commander le transducteur B jusqu'à ce que la consigne fixée pour r' soit atteinte. Dans ces conditions le transducteur $B$ peut être considéré comme une terminaison anéchoüque active.

Pour obtenir le coefficient de masquage, nous mesurons tout d'abord le déplacement du pavillon sur lequel est collé l'échantillon et ensuite la pression dans le fluide lorsque le système d'onde tend vers un système d'onde progressive. La mesure du déplacement permet de déterminer le terme $\mathrm{S}_{0}$ et la mesure directe de la pression fournit le terme $S$ de la relation (1). Le rapport direct de ces deux expressions donne le coefficient de masquage relation (4).

$$
\begin{gathered}
S_{0}=(-j) \rho c \frac{\Gamma}{\omega}=j \rho c \omega u_{0} \\
C M=\frac{P}{j \rho c \omega u_{0}}
\end{gathered}
$$

$$
\begin{aligned}
& p=\text { masse volumique de l'eau } \\
& c=\text { célérité de l'onde dans l'eau }
\end{aligned}
$$$$
\omega=\text { pulsation }
$$$$
\Gamma=\text { accélération du pavillon }
$$$$
\mathrm{u}_{0}=\text { déplacement du pavillon }
$$$$
\mathrm{P}=\text { pression mesurée derrière l'échantillon }
$$

Lors d'essais, certaines difficultés liées au comportement des transducteurs et du tube nous ont amené à étudier en détail chaque composant de cette expérience. La méthode des éléments finis avec l'aide du code ATLA a été utilisée pour expliquer ces comportements [4]. Le premier modèle, tridimensionnel, concerne le transducteur seul. Ce modèle met en évidence les deux premiers modes de flexion du transducteur à la fréquence de $844 \mathrm{~Hz}$ et de $2194 \mathrm{~Hz}$ qui perturbent effectivement la mesure.

Le second modele éléments finis creé a deux buts : le premier est de valider le principe de mesure du coefficient de masquage et le second est de comparer les résultats de ce modèle aux mesures effectués avec le tube. Le modèle ainsi validé permet de tester différentes hypothèses sur le montage de l'échantillon. La symétrie de révolution du montage expérimental a été utilisé pour réduire le nombre d'éléments. L'échantillon est parfaitement collé au pavillon du transducteur émetteur. Le champ d'onde progressive dans ce modèle est obtenu à partir de la commande en tension du transducteur récepteur. Cette tension est calculée en fonction du principe de superposition des états. L'état où le transducteur émetteur est excité à un Volt et le transducteur récepteur est en court-circuit est superposé à l'état inverse où le transducteur émetteur est court-circuité et le transducteur récepteur est excité à un Volt. La tension calculée est ensuite appliquée au transducteur récepteur. Celui-ci se comporte alors comme une terminaison anéchoïque et l'onde obtenue est quasiment progressive.

\section{RESULTATS.}

Le modèle a été utilisé dans un premier temps pour valider le principe de la mesure du coefficient de masquage avec un tube à onde progressive. Les caractéristiques du matériau employé correspondent à celles du MICROVON. Le MICROVON est une mousse de polyuréthane comportant un fort taux d'air. Ces caractéristiques acoustiques sont : $650 \mathrm{~kg} / \mathrm{m}^{3}$ pour sa masse volumique, $8.10^{6} \mathrm{~Pa}$ pour son module de Young et 0.45 pour son angle de pertes. L'épaisseur de l'échantillon est égale à $30 \mathrm{~mm}$ et son rayon correspond au rayon intérieur du tube. La figure 3 présente la comparaison entre le modèle théorique, le modele éléments finis et une mesure réalisée à la DCN/ING/SUD/LSM [5]. Le coefficient de masquage obtenu à la DCN/ING/SUD/LSM provient de la mesure des coefficients de réflexion $r$ et de transmission t autour d'un panneau de MICROVON de $30 \mathrm{~mm}$ d'épaisseur placé en cuve anéchoïque. 


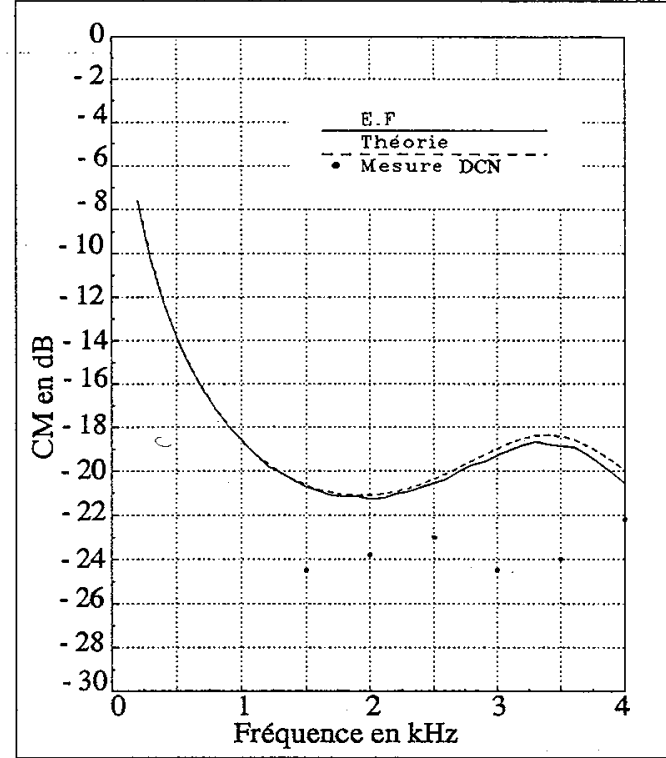

Figure 3 : Calcul et mesure du module du coefficient de masquage pour un échantillon de MICROVON (Ep. $=30 \mathrm{~mm}$ )

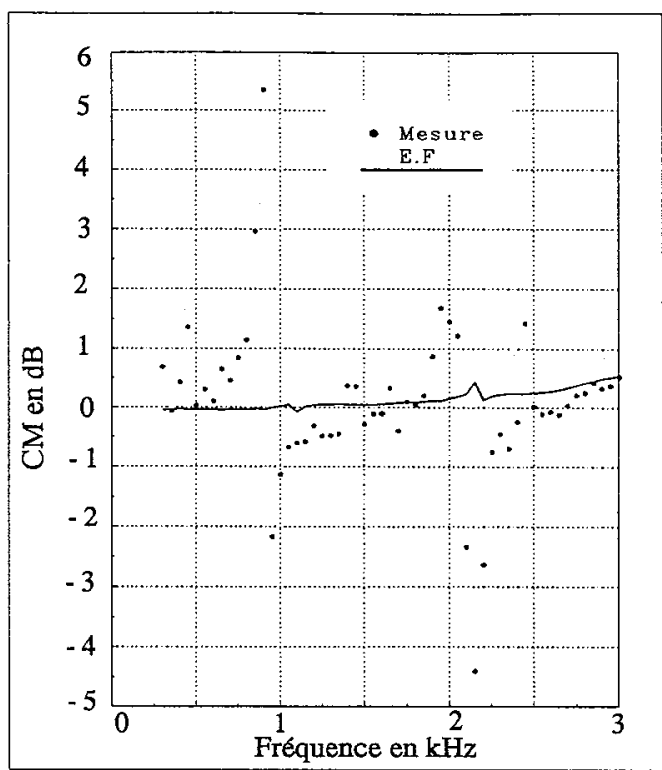

Figure 4 : Calcul et mesure du coefficient de masquage sans la présence de l'échantillon

L'écart trouvé entre le mođèle éléments finis et la mesure peut provenir d'une différence entre les caractéristiques du matériau employé dans le modèle et les caractéristiques réelles du panneau utilisé. Afin de tester expérimentalement l'équation (3) une première mesure du coefficient de masquage sans échantillon dans le tube a été effectuée. La figure 4 présente la comparaison entre le module du coefficient de masquage obtenu avec le modèle éléments finis et le coefficient mesuré.

\section{CONCLUSION.}

Le modèle éléments finis fournit un résultat en accord avec les mesures réalisées à la DCN/ING/SUD/LSM et avec le tube à onde progressive. Le modele éléments finis à cause de sa symétrie de révolution ne tient pas compte de tous les modes de résonance des transducteurs, ce qui explique les écarts rencontrés pour certaines fréquences. Avec ce modèle nous pouvons envisager l'étude de l'influence des brides de fixation entre les tronçons ainsi que celle d'un interstice fluide autour de l'échantillon sur le coefficient de masquage. Après cette étape de validation du moyen de mesure, des mesures vont être effectuées sur différents matériaux de masquage.

\section{Références.}

[1] Audoly C., "Présentation d'une méthode globale pour la caractérisation de panneaux acoustiques", Note technique N 063043, Décembre 1989. .

[2] C.Giangreco, C. Audoly," Global characterization of multilayer panels for underwater applications",Journal of wave material interactions, vol $5 \& 6 \mathrm{~N}^{\circ} 1$, January 1991.

[3] Haw G., Bigotte P., Decarpigny J.N., "Optimisation d'un banc d'étalonnage d'hydrophone à gradient de pression en basse fréquence". Rapport de synthèse, convention DRET, N 85/181, (1989).

[4] Decarpigny J.N., "Application de la méthode des éléments finis à l'étude des transducteurs piézoélectriques", Thèse de Doctorat d'Etat, Université des Sciences et Techniques de Lille, (1984).

[5] Giangreco C.,"Caractérisation de matériaux en acoustique sous-marine à l'aide de la mesure en cuve de panneaux de dimensions finies", Thèse de doctorat, Université de Compiègne, (1990). 